\title{
2-YIA-52 YIA
}

\section{Morphological changes in striatum and nucleus accumbens neurons lead to abnormal behavior in ARHGAP10 mutant mice}

\author{
Kazuhiro Hada ${ }^{1}$, Bolati Wulaer ${ }^{1}$, Taku Nagai $^{1}$, Akira Sbue $^{1}$, Masahito Sawahata ${ }^{1}$, Norimichi Itoh ${ }^{1}$, \\ Daisuke Mori ${ }^{2}$, Itaru Kushima ${ }^{2}$, Toshitaka Nabeshima ${ }^{3,4}$, Norio Ozaki ${ }^{2}$, Kiyofumi Yamada ${ }^{1}$
}

${ }^{I}$ Department of Neuropsychopharmacology and Hospital Pharmacy, ${ }^{2}$ Department of Psychiatry, Nagoya University Graduate School of Medicine, ${ }^{3}$ Advanced Diagnostic System Research Laboratory, Fujita Health University

Graduate School of Health Science, ${ }^{4}$ Aino University

Schizophrenia is a severe mental illness that affects about $1 \%$ of the population. Genetic and environmental factors contribute to the development of schizophrenia. However, the exact pathoetiology remains unclear. We generated Rho GTPase-activating protein 10 (ARHGAP10) mutant mice carrying similar variations found in Japanese schizophrenia patients. In the present study, we examined spatiotemporal expression of ARHGAP10 mRNA in the brain of mice. The expression levels of ARHGAP10 mRNA were higher in the striatum (ST) and nucleus accumbens (NAc) than those in other brain regions. We performed a series of behavior test to evaluate cognitive and emotional function in ARHGAP10 mutant mice. They showed an increase in anxiety level, and manifested potentiation of methamphetamine-induced hyperlocomotion and visual discrimination task. Morphological analysis revealed that methamphetamine-treated ARHGAP10 mutant mice showed an increase in the number of c-Fos-positive-cells in the dorsal medial striatum $(\mathrm{dmST})$ and NAc core than those in wild-type littermates. Golgi staining indicated that ARHGAP10 mutant mice showed an increase in neuronal complexity and spine density in the same brain regions compared to the wild-type mice. These results suggest that ARHGAP10 gene variations may lead to the development of cognitive and emotional deficits with morphological abnormality in the dmST and NAc core neurons. 